Original Article

\title{
Outcome of VATS Lobectomy for Elderly Non-Small Cell Lung Cancer: A Propensity Score-Matched Study
}

\author{
Xizhao Sui, MD, Hui Zhao, MD, Jun Wang, MS, Feng Yang, MD, Fan Yang, MD,
} and Yun Li, MD

\begin{abstract}
Purpose: To analyze the short-term and long-term outcome of video-assisted thoracic surgery (VATS) lobectomy for elderly patients with non-small cell lung cancer.

Methods: 105 patients aged $\geq 75$ years with resected non-small cell lung cancer were matched with 105 younger patients by propensity score. Survival rates were calculated by the Kaplan-Meier method. The cumulative incidence functions of conditional survival rate according to the age of the patients were calculated by competing risk analysis.

Results: patients $\geq 75$ years was associated with higher postoperative complication rate ( $p<0.001)$, but similar perioperative death rate $(p=0.006)$. Patients $\geq 75$ years were less likely to receive adjuvant chemotherapy $(p<0.001)$. The 5 -year overall survival rates were $54.6 \%$ for patients $\geq 75$ years and $74.1 \%$ for patients $<75$ years $(p=0.001)$. No difference was seen in disease-free survival rate $(59.5 \%$ vs. $71.9 \%$ respectively $=0.117)$. The cumulative incidence functions of 5-year cancer-specific death were similar between the two groups ( $28.7 \%$ vs. $24.6 \%$ respectively, $p=0.106$ ). The cumulative incidence functions of 5-year non-cancer-specific death was significantly higher in the elderly group $(18.7 \%$ vs. $1.0 \%, p<0.001)$.

Conclusions: VATS lobectomy for non-small-cell lung cancer in patients $\geq 75$ years were feasible with increased morbidity but similar mortality. The resected elderly patients were more frequently associated with non-cancer-specific death.
\end{abstract}

Keywords: lung cancer, VATS, lobectomy, elderly

\section{Introduction}

Anatomic pulmonary resection is the preferred radical surgery for resectable non-small cell lung cancer (NSCLC). ${ }^{1,2)}$ Lobectomy by video-assisted thoracic surgery (VATS) approach has been proved with better short-term outcomes without compromising of cancer outcomes than open surgery, ${ }^{3)}$ and theoretically is the least invasive radical intervention that more appropriate for the frail and high risk

Department of Thoracic Surgery, Center for Mini-Invasive Thoracic Surgery, People's Hospital, Peking University, Beijing, China

Received: April 22, 2015; Accepted: July 22, 2015

Corresponding author: Jun Wang. Department of Thoracic Surgery, Center for Mini-invasive Thoracic Surgery, People's Hospital, Peking

University, \#11 Xizhimen South Avenue, Beijing 100044, China

Email: jwangmd@yahoo.com

(C)2015 The Editorial Committee of Annals of Thoracic and Cardiovascular Surgery. All rights reserved. elderly population. However, the benefit of radical surgical treatment may decrease with age due to reduced life expectancy and reduced tolerance of stress in the elderly population. And relatively few studies have analyzed in detail the cancer outcome of elderly patients underwent VATS lobectomy. We decided to perform a propensity score-matched study analyzing the short-term and long-term outcome in elderly patients undergoing lobectomy to figure out if VATS lobectomy afforded the similar cure effect on elderly lung cancer patients as the general population.

\section{Material and Methods}

\section{Patients}

We conducted a retrospective review of data on patients with non-small cell lung cancer who underwent VATS anatomic major pulmonary resection from September 2006 to October 2013 in Peking University People's 
Hospital, Beijing, China. Patients with small-cell lung cancer, tumor in situ, stage III/IV, neoadjuvant therapies, and incomplete resections were excluded. And 908 patients with clinical stage I/II NSCLC were identified with 105 patients aged $\geq 75$ year. A propensity score-matched analysis was performed for matching the elderly patients to the 105 control subjects aged $<75$ years by five values which we considered might impact the outcome the radical lobectomy. The values were as follows: Gender, pathology, stage, type of pulmonary resection and year of operation.

The following variables were analyzed: patient characteristics including age, gender, smoke index, body mass index (BMI), pulmonary function test (forced expiratory volume in 1 second (FEV1), forced vital capacity rate of one second (FEV1.0/FVC), transfer factor of lung for carbon monoxide (TLCO)), comorbidity, cancer site, pathology, stage, perioperative data including type of pulmonary resection procedure, year of operation, converted to thoracotomy, operation time, bleeding, drainage time, postoperative stay, red blood cell (RBC) transfusion, complication, and perioperative death. Smoke index was calculated as years of smoking multiplied by cigarettes per day. Comorbidity (type and number) was collected according to an adapted version of the Charlson index. ${ }^{4)}$ All the lung cancers were staged pathologically according to the IASLC Seventh Edition of the TNM Classification. The perioperative complication and perioperative death was defined as any complication or death within 30 days of the operation. The database for patients resected by thoracoscopic lobectomy in our institution was approved by our Institutional Review Board in 2011, collecting retrospective data between 2006 and 2010, and prospective data since 2011. This study was also approved by the Institutional Review Board.

All patients were followed up for every six months. Follow-up information including status of adjuvant chemotherapy, date of recurrence, recurrence location, date of death, and cause of death. Recurrence-free status was censored at the last follow-up visit. Follow-up information was complete through December 2014.

\section{Surgical method}

All patients were on one-lung ventilator with a double lumen endotracheal tube under general anesthesia. Thoracoscopic lobectomy was performed by three ports. The observation hole was located at the midaxillary line of the 7th or 8th interspace, the main operating hole was located at the anterior axillary line of the 4th interspace for upper lobectomies or the 5th interspace for middle and lower lobectomies with a length of 3-4 cm, while the auxiliary incision was at the infrascapular line of the 7th or 8th interspace. The sequence of thoracoscopic lobectomy was basically similar to that of conventional lobectomy performed via thoracotomy. Systemic lymph node dissection was performed after lobectomy.5) In situations when thoracotomy was converted, incisions were extended $12-15 \mathrm{~cm}$ towards the lower angle of scapula, and the lobectomy and lymph node dissection was completed under direct visualization.

\section{Statistical analysis}

All the statistical analysis were carried out using SPSS 18.0 (IBM SPSS statistics, IBM Corp.) and the R 3.1.1 software package (http://www.r-project.org). The normality test of measurement data was conducted by using Kolmogorov-Smirnov Test. Normality data between the two groups of patients were compared by independentsamples $\mathrm{T}$ test, and Non-normal data were compared by two sample rank test. Chi-square tests were applied for analysis of categorical data respectively. Survival rates were calculated by the Kaplan-Meier estimated survival curves. Statistical comparisons between curves were performed using the log-rank test. The cumulative incidence function (CIF) of the cancer-specific death was computed according to the age of the NSCLC patients (elderly and younger), adjusting for non-cancer-specific death as a competing risk. The CIF was estimated using the cmprsk package in R (Gray, 1988; Kim, 2007). A P-value was considered statistically significant.

\section{Results}

\section{Patient characteristics}

The median age of the elderly group and the control were 78.0 vs. 60.2 respectively, with a majority of male, adenocarcinoma, stage IA and IB. The basic characteristics of the two groups were compared in Table 1. The elderly group had worse BMI, American Society of Anaesthesiologists (ASA) grade, FEV1, FEV1/FVC, and TLCO $\%(\mathrm{p}<0.05)$. Two third $(66.7 \%)$ of the elderly patients had at least one comorbidity, and so were $50.5 \%$ of the younger. The elderly group also had increased number of comorbidities comparing with the control $(\mathrm{p}$ $=0.042$ ). We found no statistically significant difference in the rate of malignancy history in the elderly group and the control $(\mathrm{p}=0.298)$. The mean follow-up times were 29.1 months in the elderly group and 34.5 months in the younger group $(\mathrm{p}=0.723)$ with the rates of loss to follow up of $3.8 \%$ and $1.9 \%$ respectively $(\mathrm{p}=0.403)$. 
Table 1 Patients characteristics

\begin{tabular}{|c|c|c|c|}
\hline Variable & Elderly & Control & $\mathrm{p}$ value \\
\hline \multicolumn{4}{|l|}{ Patient characteristics } \\
\hline \multicolumn{4}{|l|}{ Gender } \\
\hline Male & 72 & 73 & 0.881 \\
\hline Female & 33 & 32 & \\
\hline Age & $78.0 \pm 2.7$ & $60.2 \pm 9.4$ & $<0.001$ \\
\hline Smoke index & 333.9 & 319.8 & 0.807 \\
\hline $\mathrm{BMI}^{\mathrm{a}}$ & $24.7 \pm 6.8$ & $28.1 \pm 7.3$ & 0.001 \\
\hline $\mathrm{ASA}^{\mathrm{b}}$ & $2.12 \pm 0.41$ & $1.87 \pm 0.48$ & $<0.001$ \\
\hline \multicolumn{4}{|l|}{ Comorbidity (no.) } \\
\hline 0 & 35 & 52 & \\
\hline 1 & 46 & 31 & 0.042 \\
\hline$\geq 2$ & 24 & 22 & \\
\hline Malignancy & $6(5.7 \%)$ & $10(9.5 \%)$ & 0.298 \\
\hline FEV $1^{c}$ & $2.06 \pm 0.48$ & $2.56 \pm 0.73$ & $<0.001$ \\
\hline FEV1/FVC $(\%)^{\mathrm{d}}$ & $74.45 \pm 9.58$ & $76.95 \pm 8.44$ & 0.032 \\
\hline $\operatorname{TLCO}(\%)^{\mathrm{e}}$ & $75.12 \pm 17.16$ & $84.94 \pm 15.89$ & $<0.001$ \\
\hline \multicolumn{4}{|l|}{ Cancer site } \\
\hline RUL & 33 & 37 & \\
\hline RML & 15 & 9 & \\
\hline RLL & 21 & 18 & 0.665 \\
\hline LUL & 18 & 19 & \\
\hline LLL & 18 & 22 & \\
\hline \multicolumn{4}{|l|}{ Pathology } \\
\hline Adenocarcinoma & 75 & 74 & 0.492 \\
\hline Squamous cell carcinoma & 27 & 29 & \\
\hline Adenosquamous & 1 & 2 & \\
\hline Large cell carcinoma & 2 & 0 & \\
\hline \multicolumn{4}{|l|}{ Stage } \\
\hline IA & 29 & 29 & \\
\hline IB & 41 & 40 & \\
\hline IIA & 15 & 11 & 0.445 \\
\hline IIB & 0 & 3 & \\
\hline IIIA & 20 & 22 & \\
\hline
\end{tabular}

${ }^{\mathrm{a} B M I}$ : body mass index; ${ }^{\mathrm{b}} \mathrm{ASA}$ : The American Society of Anesthesiologists physical status classification system; ${ }^{\mathrm{C}} \mathrm{FEV} 1$ : forced expiratory volume in one second; ${ }^{\mathrm{d}} \mathrm{FEV} 1 / \mathrm{FVC}$ : forced vital capacity rate of one second; ${ }^{\mathrm{T}} \mathrm{TLCO}$ : transfer factor of the lung for carbon monoxide; RUL: right upper lobe; RML: right middle lobe; RLL: right lower lobe; LUL: left upper lobe; LLL: left lower lobe

\section{Short-term results of the patients}

Table 2 compared the perioperative data of the two groups, and demonstrated no significant difference in operative time, blood loss, rate of converted thoracotomy between the two groups. However, the length of drainage duration and postoperative stay in the elderly group were increased than the controls $(\mathrm{p}<0.05)$. The elderly group was also with higher rate of postoperative complication ( $41.9 \%$ vs. $14.3 \%, \mathrm{p}<0.001)$, RBC transfusion $(14.3 \%$ vs. $3.9 \%, p=0.014)$. The rate of perioperative death was also increased, but was not significantly different (4.5\% vs. $0, p=0.06)$ across groups. Fewer patients in elderly group received further adjuvant chemotherapy (13.3\% vs. $49.5 \%, \mathrm{p}<0.001)$.

\section{Long-term survival of the patients}

Mean follow-up time of the study was 28 months. Figure 1 gives the details of the disease-free survival curve and overall survival by Kaplan-Meier method. The elderly had a worse 5-year overall survival rate than controls, with $54.6 \%$ and $74.1 \%$ respectively $(\mathrm{p}=0.001)$. No difference was seen in disease-free survival rate, with 5 -year rate of $59.5 \%$ and $71.9 \%$ respectively for the elderly and the younger groups $(\mathrm{p}=0.117)$. 
Table 2 Perioperative data

\begin{tabular}{lccc}
\hline Variable & Elderly & Control & p value \\
\hline Procedure & & & 0.250 \\
Lobectomy & 100 & 101 & \\
Bilobectomy & 5 & 2 & \\
Sleeve lobectomy & 0 & 1 & \\
$\quad$ Pneumonectomy & 0 & 1 & \\
Converted to thoracotomy & $9(8.6 \%)$ & $14(13.3 \%)$ & 0.269 \\
Operation time & $195.7 \pm 56.9$ & $202.7 \pm 69.5$ & 0.786 \\
Bleeding & $194.3 \pm 251.7$ & $193.3 \pm 372.8$ & 0.123 \\
Drainage time & $7.52 \pm 3.51$ & $6.06 \pm 3.13$ & 0.001 \\
Postoperative stay & $10.66 \pm 4.91$ & $8.38 \pm 3.44$ & $<0.001$ \\
Transfusion & $15(14.3 \%)$ & $4(3.8 \%)$ & 0.014 \\
Complication & $44(41.9 \%)$ & $15(14.3 \%)$ & $<0.001$ \\
Perioperative death & $5(4.8 \%)$ & 0 & 0.06 \\
Adjuvant chemotherapy & $14(13.3 \%)$ & $52(49.5 \%)$ & $<0.001$ \\
\hline
\end{tabular}
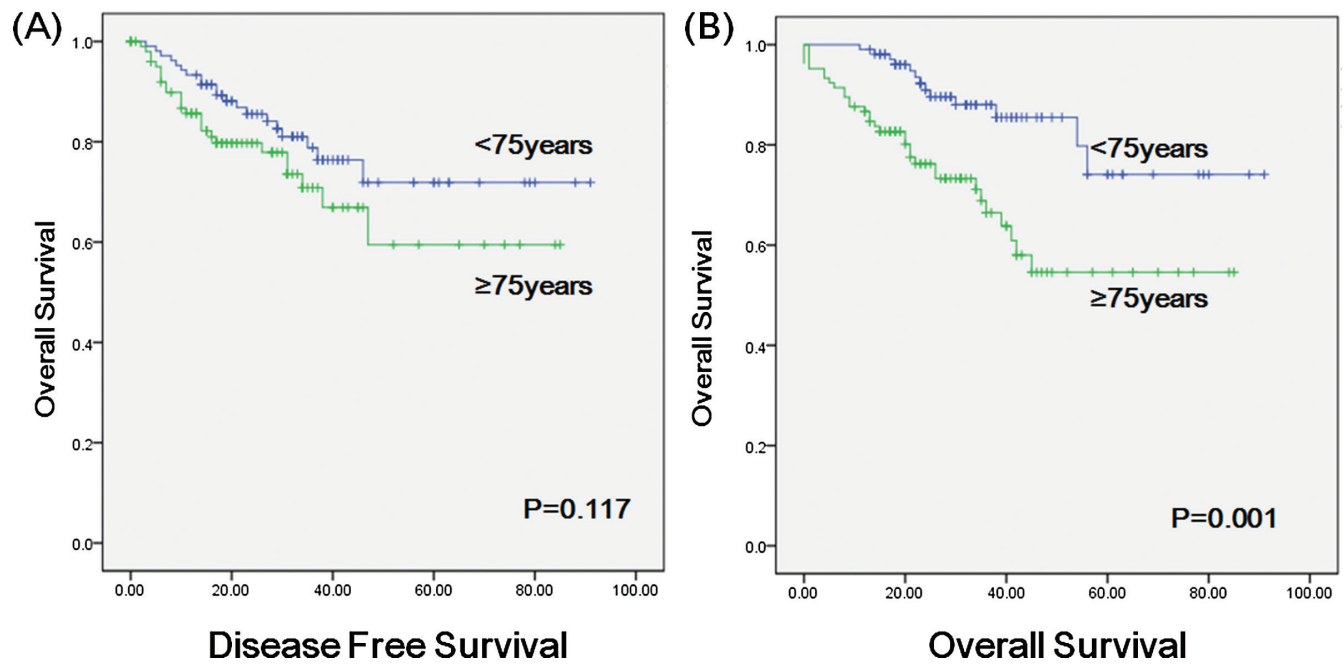

Fig. 1 Disease-free survival (A) and overall survival (B) for the elderly and younger groups.

Figure 2 illustrates further the relationship between age and the cancer-specific death, taking the competing risk of non-cancer-specific death into account. The CIFs of cancer-specific death were not significantly different between the two groups with 5-year cancer-specific death rate of $28.7 \%$ and $24.6 \%$ respectively $(\mathrm{p}=0.106)$. However, the elderly group was significantly associated with the incidence of non-cancer-specific death vs. the control $(18.7 \%$ vs. $1.0 \%, \mathrm{p}<0.001)$.

\section{Discussion}

Lung cancer is a disease of the elderly. Data from Surveillance, Epidemiology, and End Results (SEER) revealed about $71 \%$ of newly diagnosed cases occur in patients aged over 70 years, more than one third of them was 80 years or older in 2005-2009. $\left.{ }^{6}\right)$ It is a very relevant but also understudied question whether a curative resection is needed for this increasing population of elderly patients presenting with early-stage lung carcinoma. Elderly patients with early stage lung cancer were more likely to have poor performance status and multiple comorbidities leading to less accurate staging and were not willing or able to undergo surgical intervention. ${ }^{7,8)}$ As demonstrated in this study, the elderly were indeed in a more fragile background than the younger patients although they were all carefully selected specially for good cardiopulmonary function that meet the operative criteria for radical lobectomy. Even if lobectomy by VATS for the elderly was recommended more commonly now, 
(A)

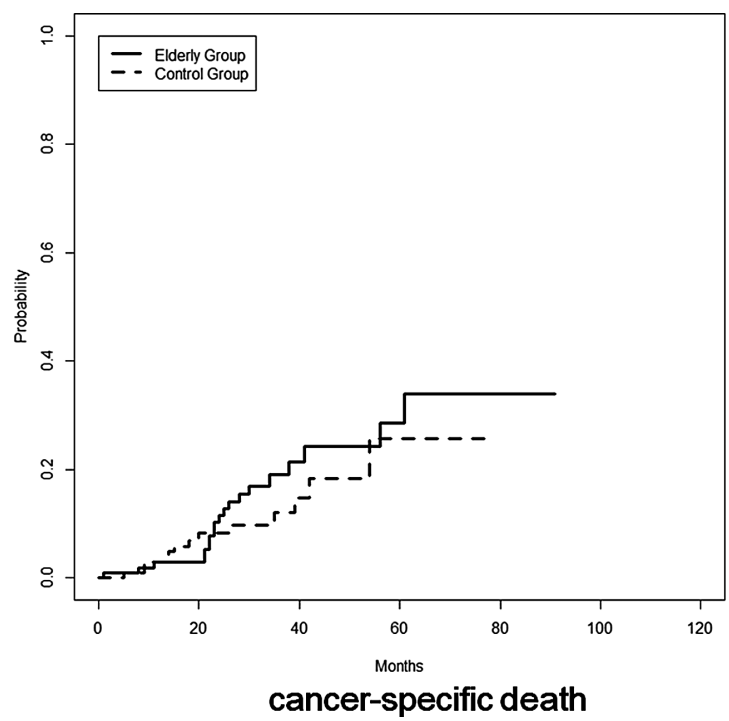

(B)

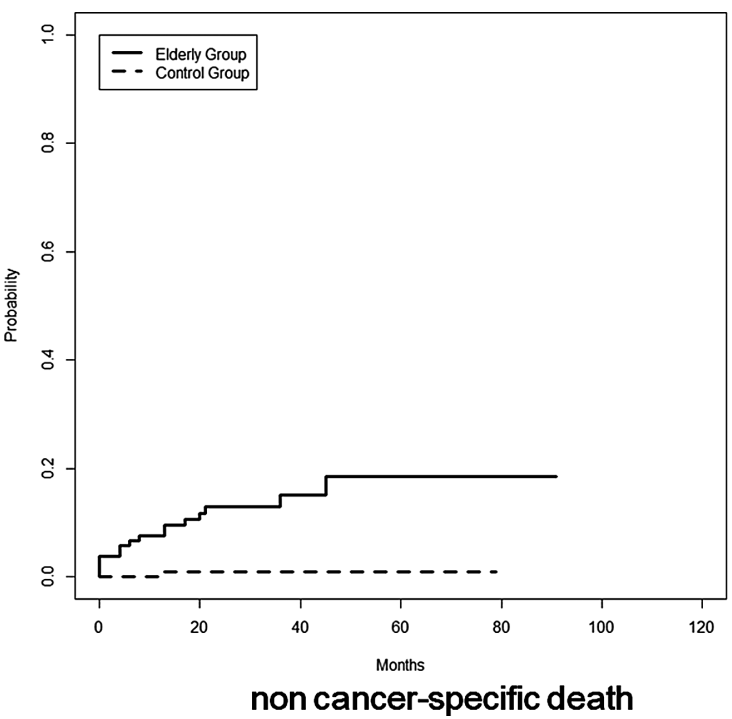

Fig. 2 The cumulative incidence function of cancer-specific death (top, A) and non-cancer-specific death (bottom, B) for the elderly and younger groups.

these characteristics were still risky factors that might be associated with potentially higher complications. ${ }^{9)}$

The data about the relationship between ages and the outcome of open lobectomy for elderly NSCLC patients were conflicting in studies with various definitions of "elderly". Suemitsu et al. also reported elderly patients more frequently had perioperative complications but similar mortality than younger patients ${ }^{10}$. While Rueth et al. found that postoperative complications were significantly associated with age at least 75 years for older patients undergoing lobectomy for stage I non-small cell lung cancer. ${ }^{9)}$ In contrary to these studies, others reported in selected fit elderly patients, radical lobectomy is tolerable and is associated with survival similar to that of younger patients. ${ }^{6,11)}$ VATS approach to lobectomy in the elderly could achieve better short-term outcome compared with thoracotomy. ${ }^{12)}$ Nevertheless, there was no significant difference in the mortality rate in elderly who were associated with significantly higher complications compared with the control in this study. We ascribed to the multidisciplinary consultation and the manageability of the majority of the complications. There were five perioperative death among the elderly patients. The reason were as follows: two ARDS, two pulmonary embolisms and one cardiac infarction, which were all unpredictable urgent and critical complications. The perioperative death ratio of the general population in our institution was less than $0.5 \%$. No case of perioperative death was matched into the analysis in the younger group. Based on the results in this study, we thought the short-term outcome of VATS lobectomy for elderly NSCLC was acceptable, but it should always be reminded about the potential risk of the sudden "disastrous" events postoperatively.

Major pulmonary resection removed the entire cancerinvolved lobe and offered the best chance of long-term cure for the resectable lung cancer patients. And it is now widely accepted that the surgical treatment of NSCLC in the elderly is feasible and that age per se is not a contraindication for various surgical procedures. ${ }^{13)}$ However, the main findings of this study showed that the radical lobectomy achieved similar disease-free survival but failed in overall survival for the elderly lung cancer patients comparing with the younger patients. The similar results were reported by Kamiyoshihara. ${ }^{14)}$ We use competing risk method to analyze the relationship between age and the death cause. We admitted that the information on cancer-related death is usually very limited because of lacking information, and due to autopsy being performed in few cases. So we supposed any patients with cancer recurrence but uncertain death cause as cancer-specific death in this study. Our further analysis by competing risk method revealed similar cancer-specific survival between the elderly and the control. Nevertheless, the estimated CIF of non-cancer-specific survival revealed that one fifth of the elderly just died before the recurrence of the primary tumor. These results suggested that the oncologic outcomes of resected lung cancer 
were not age-related. Schneider found that an impaired performance status had a significant negative impact on survival in subjects $>75$ years. ${ }^{15)}$ We think the fragility and poorer life expectation of the elderly in this study were the contributing factor so that the radical lobectomy failed to achieve the cancer benefit for the elderly as for the control. They just died early after the radical intervention no matter if the tumors relapsed. Another potential contributing factor, the lower ratio of adjuvant chemotherapy adoption in the elderly and subsequent salvage treatment after recurrence compared with the control might impact the survival benefic as well. ${ }^{16)}$

The high incidence of non-cancer related death of the elderly NSCLC patients in this study maybe as a result of the inadequacy of comprehensive assessment of the frailty of the elderly. Preoperative Assessment of Cancer (PACE) incorporating Comprehensive Geriatric Assessment (CGA), brief fatigue inventory, performance status, and ASA grade was further developed to determine the suitability of elderly surgical candidate. ${ }^{17)}$ The PACE had been established by a prospective study of International Society of Geriatric Oncology (SIOG) as a valuable tool in enhancing surgical decision making concerning the candidacy of elderly cancer patients for surgical intervention. ${ }^{18)}$ And it was now recommended to be used routinely in surgical practice. These tools were effective to ensure that appropriate treatment choice was guided by patient characteristics and not by age. However, in this retrospective study, few data are available for the comprehensive scale assessment. So we cannot figure out the quantifiable data about the fragility of the elderly in this study. Meanwhile, we noticed that few data available when searching about CGA or PACE especially on elderly NSCLC surgical candidates. ${ }^{19)}$ Different instruments have been valid to encourage oncologists for screening older cancer patients for further a CGA. ${ }^{20)}$ So we think it is urgent to emphasize the perioperative comprehensive assessment of aging but not only fulfill the common criteria of lobectomy.

Sublobar resection for the frail elderly patients seemed to be a better choice that balanced the short-term surgical risk and the long-term cancer benefit. The only randomized clinical trial from Lung Cancer Study Group denied sublobar resection as the radical surgical procedure of choice with peripheral T1 N0 non-small cell lung cancer because of the higher death rate and locoregional recurrence rate. ${ }^{21)}$ Nevertheless, subsequent two large retrospective studies reported that sublobar resection conferred similar long-term survival versus lobectomy although it had been associated with a higher locoregional recurrence rate in patients aged $\geq 75$ years. ${ }^{22,23)}$ The significant survival benefit of lobectomies over sublobar resection is not evident for patients $>71$ years of age. ${ }^{22)}$ Currently there are two randomized controlled trials ongoing with the respect to sublolar resection for less advanced NSCLC, CALGB14050324) in US and JCOG0802 ${ }^{25}$ in Japan. The indications of both trials focus on very early stage NSCLC with diameter $\leq 2 \mathrm{~cm}$ to investigate the curable utility of sublobar resection. However, elderly patients may be under-represented in both trials. We suggest a randomized control trial focused on the elderly NSCLC patients is necessary to confirm the noninferiority of sublobar resection versus standard lobectomy in this population, with a more extended indication comparing the randomized trials above said.

This study investigated an important and timely issue: whether lobectomy by minimally invasive VATS approach could benefit the elderly population of lung cancer patients as in the general population. This was a retrospective study from a relatively smaller database in a single institution, and propensity score-matching was used attempted to eliminate selection bias. However, there were some limitations in this study. First, we did not exclude patients who had an initial VATS and was converted to thoracotomy ( $8.6 \%$ vs. $13.3 \%, p=0.269)$. Though this might be related with increased complications. But we thought this would reflex the result of the surgery decision as it was not the real that we chose to perform VATS lobectomy for the elderly unless we were sure thoracotomy were not needed. Besides, the converted rate between the two groups were comparable and therefore did not bias the results. Second, this is still a retrospective analysis, and there may be issues could not be discussed due to missing data. Such as quality of life, Sullivan et al. reported elderly patients undergoing lobectomy for NSCLC had similar pulmonary function test (PFT) results and functional status at 1 year postoperatively. ${ }^{11)}$ We just did not have retrospective data to comparing quality of life between the two groups. Third, as said above, the data do not conclusively indicate the relationship between the fragility of the patients and the surgical outcome due to lack of geriatric assessment data.

\section{Conclusions}

VATS lobectomy for non-small-cell lung cancer in patients $\geq 75$ years were feasible with increased morbidity but similar mortality. However, elderly patients were more frequently associated with non-cancer-specific death. Radical resection of lung cancer in elderly patients should be decided personalized. 


\section{Disclosure Statement}

All authors disclose no financial and personal relationships with other people or organizations that could inappropriately influence our work.

\section{References}

1) Scott WJ, Howington J, Feigenberg S, et al. Treatment of non-small cell lung cancer stage I and stage II: ACCP evidence-based clinical practice guidelines (2nd edition). Chest 2007; 132: 234S-242S.

2) Boffa DJ, Allen MS, Grab JD, et al. Data from The Society of Thoracic Surgeons General Thoracic Surgery database: the surgical management of primary lung tumors. J Thorac Cardiovasc Surg 2008; 135: 247-54.

3) Paul S, Altorki NK, Sheng S, et al. Thoracoscopic lobectomy is associated with lower morbidity than open lobectomy: a propensity-matched analysis from the STS database. J Thorac Cardiovasc Surg 2010; 139: 366-78.

4) Charlson ME, Pompei P, Ales KL, et al. A new method of classifying prognostic comorbidity in longitudinal studies: development and validation. J Chronic Dis 1987; 40: 373-83.

5) Wang J, Li Y, Liu J, et al. Completely thoracoscopic lobectomy for early stage non-small-cell lung cancer. Chin J Thorac Cardiovasc Surg 2008; 24: 147-50.

6) Cerfolio RJ, Bryant AS. Survival and outcomes of pulmonary resection for non-small cell lung cancer in the elderly: a nested case-control study. Ann Thorac Surg 2006; 82: 424-9; discussion 429-30.

7) Janssen-Heijnen ML, Schipper RM, Razenberg PP, et al. Prevalence of co-morbidity in lung cancer patients and its relationship with treatment: a population-based study. Lung Cancer 1998; 21: 105-13.

8) Endoh H, Yamamoto R, Satoh Y, et al. Risk analysis of pulmonary resection for elderly patients with lung cancer. Surg Today 2013; 43: 514-20.

9) Rueth NM, Parsons HM, Habermann EB, et al. Surgical treatment of lung cancer: predicting postoperative morbidity in the elderly population. J Thorac Cardiovasc Surg 2012; 143: 1314-23.

10) Suemitsu R, Takeo S, Hamatake M, et al. The perioperative complications for elderly patients with lung cancer associated with a pulmonary resection under general anesthesia. J Thorac Oncol 2009; 4: 193-7.

11) Sullivan V, Tran T, Holmstrom A, et al. Advanced age does not exclude lobectomy for non-small cell lung carcinoma. Chest 2005; 128: 2671-6.

12) Cattaneo SM, Park BJ, Wilton AS, et al. Use of videoassisted thoracic surgery for lobectomy in the elderly results in fewer complications. Ann Thorac Surg 2008; 85: 231-5; discussion 235-6.

13) Pallis AG, Gridelli C, Wedding U, et al. Management of elderly patients with NSCLC; updated expert's opinion paper: EORTC Elderly Task Force, Lung Cancer Group and International Society for Geriatric Oncology. Ann Oncol 2014; 25: 1270-83.

14) Kamiyoshihara M, Kawashima O, Ishikawa $S$, et al. Long-term results after pulmonary resection in elderly patients with non-small cell lung cancer. J Cardiovasc Surg (Torino) 2000; 41: 483-6.

15) Schneider T, Pfannschmidt J, Muley T, et al. A retrospective analysis of short and long-term survival after curative pulmonary resection for lung cancer in elderly patients. Lung Cancer 2008; 62: 221-7.

16) Cuffe S, Booth CM, Peng Y, et al. Adjuvant chemotherapy for non-small-cell lung cancer in the elderly: a population-based study in Ontario, Canada. J Clin Oncol 2012; 30: 1813-21.

17) Pope D, Ramesh H, Gennari R, et al. Pre-operative assessment of cancer in the elderly (PACE): a comprehensive assessment of underlying characteristics of elderly cancer patients prior to elective surgery. Surg Oncol 2006; 15: 189-97.

18) PACE participants, Audisio RA, Pope D, et al. Shall we operate? Preoperative assessment in elderly cancer patients (PACE) can help. A SIOG surgical task force prospective study. Crit Rev Oncol Hematol 2008; 65: 156-63.

19) Gironés R, Torregrosa D, Maestu I, et al. Comprehensive Geriatric Assessment (CGA) of elderly lung cancer patients: A single-center experience. J Geriatr Oncol 2012; 3: 98-103.

20) Owusu C, Koroukian SM, Schluchter M, et al. Screening older cancer patients for a Comprehensive Geriatric Assessment: A comparison of three instruments. J Geriatr Oncol 2011; 2: 121-9.

21) Ginsberg RJ, Rubinstein LV. Randomized trial of lobectomy versus limited resection for T1 N0 non-small cell lung cancer. Lung Cancer Study Group. Ann Thorac Surg 1995; 60: 615-22; discussion 622-3.

22) Mery CM, Pappas AN, Bueno R, et al. Similar longterm survival of elderly patients with non-small cell lung cancer treated with lobectomy or wedge resection within the surveillance, epidemiology, and end results database. Chest 2005; 128: 237-45.

23) Okami J, Higashiyama M, Asamura H, et al. Pulmonary resection in patients aged 80 years or over with clinical stage I non-small cell lung cancer: prognostic factors for overall survival and risk factors for postoperative complications. J Thorac Oncol 2009; 4: 1247-53.

24) Altorki N, Keenan R, Bauer T, et al. CALGB 140503/ Endorsed Study: "A Phase III Randomized Trial of Lobectomy Versus Sublobar Resection For Small $(<2 \mathrm{Cm})$ Peripheral Non-Small Cell Lung Cancer". http://www.cancer.gov/clinicaltrials/CALGB-140503.

25) Nakamura K, Saji H, Nakajima R, et al. A phase III randomized trial of lobectomy versus limited resection for small-sized peripheral non-small cell lung cancer (JCOG0802/WJOG4607L). Jpn J Clin Oncol 2010; 40: 271-4. 